\title{
New basis for the constitutive modelling of aggregated soils
}

\author{
Azad Koliji · Laurent Vulliet · Lyesse Laloui
}

Received: 18 April 2007 / Accepted: 7 December 2007/Published online: 19 February 2008

(C) Springer-Verlag 2008

\begin{abstract}
Natural and compacted soils are usually characterized by aggregation of particles. The mechanical behaviour of these materials depends on soil structure. The oedometric compression tests performed on aggregated samples presented here showed that these materials exhibit a yield limit depending not only on stress history and stress state but also on soil structure. Evidence is provided using the neutron tomography technique. These results revealed that soil structure modification occurs together with plastic deformations. The experimental results are used to propose a new state parameter to quantify the soil structure. Based on pore-scale experimental observations, an evolution law for this parameter is proposed as a function of associated plastic strains. Considering both soil fabric and inter-particle bonding effects, a new yield limit depending on stress state, stress history and soil structure is introduced for the aggregated soils. Accordingly, a new constitutive framework consistent with strain hardening plasticity is proposed to consider soil structure effects in the modelling of aggregated soils.
\end{abstract}

Keywords Aggregated soil - Constitutive modelling . Double porosity $\cdot$ Neutron tomography $\cdot$ Soil structure

\section{List of symbols}

$e^{\mathrm{m}} \quad$ Macrovoid ratio

$n, n^{\mu}, n^{\mathrm{m}} \quad$ Total, micro- and macroporosity

$p_{\mathrm{c}}{ }^{\prime} \quad$ Apparent preconsolidation pressure

\footnotetext{
A. Koliji $(\bowtie) \cdot$ L. Vulliet · L. Laloui

Soil Mechanics Laboratory, Ecole Polytechnique Fédérale de Lausanne (EPFL), EPFL-ENAC-ICARE-LMS, Station 18, 1015 Lausanne, Switzerland

e-mail: azad.koliji@epfl.ch
}
$p_{\mathrm{c}}{ }^{*}$
Intrinsic preconsolidation pressure corresponding to reconstituted soil
$R \quad$ Degree of soil structure
$\varepsilon^{\mathrm{D}} \quad$ Destructuring strain
$\Psi^{\text {st }} \quad$ Soil structure function
$\omega \quad$ Rate controlling parameter for structure degradation

\section{Introduction}

A rigorous description of the mechanical behaviour of soils, as a material with hierarchical levels of heterogeneity, demands a comprehensive understanding of soil structure and its evolution during loading. The term soil structure represents the combination of soil fabric, i.e. arrangement of particles, and inter-particle bonding [18]. Although any soil possesses a particular structure, soil structure effects are particularly observed in soils referred to as structured soils. Fabric of these materials is commonly subjected to aggregation of particles. The size of aggregates depends on several factors and might differ by five orders of magnitude from the clay platelets in compacted clays to large aggregates and clods in natural and agricultural soils. These materials are characterized by a double porosity fabric $[8,10,14]$. Accordingly, two levels of structure can be identified in an aggregated soil: the soil microstructure, defined as the elementary particle associations within the soil aggregates, and the macrostructure, corresponding to the arrangement of these soil aggregates and the relation among the structural units [1]. Experimental observation of these two levels of structures are shown in Fig. 1. Figure 1a shows a three-dimensional aggregated sample of silty clay obtained by neutron 

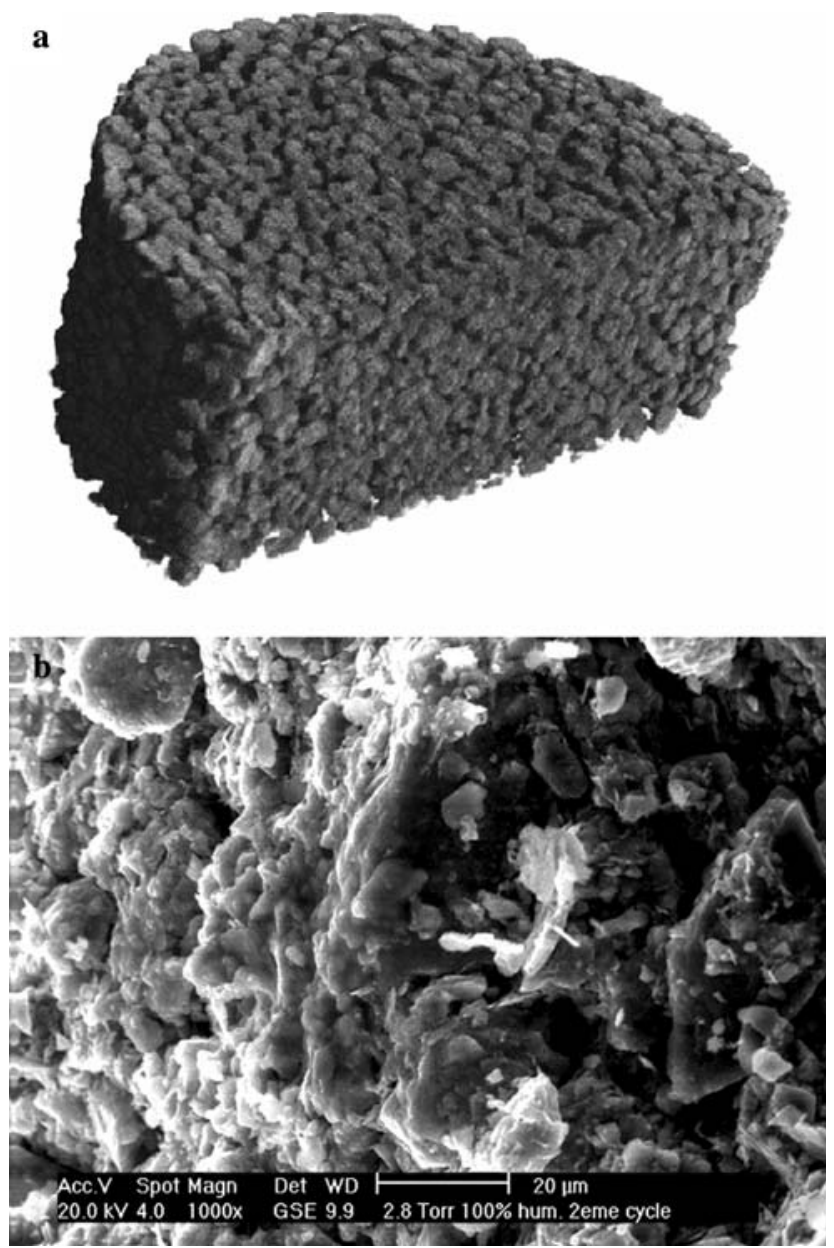

Fig. 1 Two levels of structure in aggregated Bioley silt. a Macropores and aggregates in a 3D volume of the sample half $(35 \mathrm{~mm}$ in height $\times 80 \mathrm{~mm}$ in diameter) obtained by neutron tomography, b Micropores and soil particles in a single aggregate obtained by environmental scanning electron microscopy (picture size $120 \times 90 \mu \mathrm{m})$

tomography where the aggregates and the macropores between them represent the macrostructure of the soil. Figure $1 \mathrm{~b}$ shows a $1,000 \times$ magnified image of a single aggregate of the same soil using an environmental scanning electron microscope. This figure illustrates the existence of micropores within individual aggregates which correspond to the microstructure of the soil. Any deformation affecting these materials should be considered as a combined phenomenon at both levels of soil structure.

Over the last few years, new experimental studies have led to a better characterization of the macroscopic mechanical behaviour of natural bonded soils $[6,17]$ and, simultaneously, to several improvements in constitutive models $[4,12,19,20]$. These works mainly focus on the inter-particle bonding effects. Other studies have included fabric effects in constitutive models for compacted expansive clays $[9,21]$. Due to the difficulty of obtaining microscale experimental proof, research work on this topic is, however, largely based on hypothesis rather than on experimental evidence.

This study presents an original contribution to the field of constitutive modelling of aggregated soils. Aggregated soils are structured soils with double porosity in which both fabric and inter-particle bonding effects should be considered in a single constitutive framework. In the first part of the paper, the mechanical behaviour of soil at the macro scale and soil structure evolution at the pore scale during compression are investigated based on experimental evidence. The rest of the paper is aimed at introducing the soil structure parameters and to proposing a framework for including soil structure effects in constitutive models of soils based on strain hardening plasticity.

\section{Experimental evidences}

In the first part of the experimental study, oedometric compression tests were performed on both aggregated and reconstituted soils in order to characterize the influence of soil structure on the macroscopic behaviour of the material. These tests were performed on two types of aggregated clayey silts, namely Abist and Bioley. Abist soil is a silty clay from central Switzerland with a liquid limit, $w_{1}$, of $38.4 \%$ and a plasticity index, $I_{\mathrm{p}}$, of $17.7 \%$. These values for the Bioley soil are $28.9 \%$ and $13.4 \%$, respectively. After sampling in the field, the Abist soil was first gently crushed into smaller parts at its own natural water content of about $7 \%$. The visible organic parts were then carefully removed and aggregates with an average size of $4 \mathrm{~mm}$ were selected by sieving. In the case of the Bioley silt, aggregates were artificially prepared from the reconstituted soil. The soil was first compacted at water content of $13 \%$, which is slightly on the dry side of optimum (13.7\%), then gently crushed into smaller clods. Similar to the Abist soil, the aggregates were then selected by sieving. In both soils, the obtained aggregates were subjected to oven drying for $48 \mathrm{~h}$ prior to testing. For each soil, the reconstituted material was obtained by crushing and remoulding of the soil.

In the second part of the experimental program, neutron tomography was used to evaluate the soil structure evolution in a dry aggregated sample of Abist soil subjected to oedometric compression.

\subsection{Oedometric response}

Oedometric compression tests under dry condition were performed on both aggregated and reconstituted samples of both soils. Aggregated samples were prepared by free placement of dry aggregates in the cell followed by a very 
slight compaction. This method of preparation yields a sample exhibiting large macropores with a relatively high initial void ratio. Dry reconstituted samples were also prepared directly in the oedometric cell without major compaction prior to the test. Since the samples were dry, the required time of equilibrium for loading step were quite short and the test could be carried out in maximum 3 days.

Analogous results were observed for both the Bioley and Abist soils. Figure 2 illustrates the oedometric response of the aggregated and reconstituted samples of both the Bioley and Abist soil. Although the aggregated sample was not previously consolidated, an initial stiff behaviour followed by yielding can be observed in its oedometric compression response. This behaviour could be described as an induced overconsolidation, although with a different origin stemming from the soil structure. Therefore, the corresponding yield stress is referred to, here, as the apparent preconsolidation stress. Accordingly, the apparent preconsolidation stress in these aggregated materials depends not only on stress history and stress state, but also on the soil structure. Another piece of evidence observed in Fig. 2 is the fact that the compression curve of the aggregated soil is located to the right side of the normal compression curve of the reconstituted soil. As expected, at a given value of applied stress, the aggregated soil exhibited a higher void ratio in comparison with the reconstituted soil.

Figure 2 also illustrates that the compression curves of the aggregated and reconstituted soils tend to converge at higher values of applied stress. It can be seen that the virgin oedometric compression line of the reconstituted Bioley soil is an asymptote to the compression curve of the

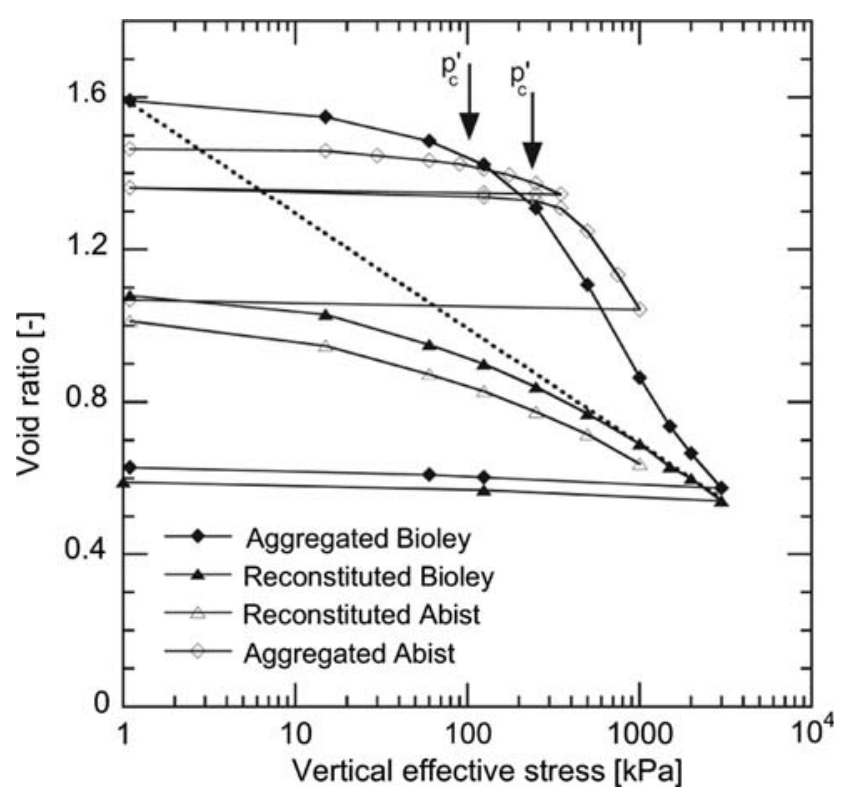

Fig. 2 Oedometric response of aggregated and reconstituted Bioley silt aggregated soil that intersects the curve at the initial virgin state (dashed line). The slope of the initial compression, i.e., apparently overconsolidated part, in aggregated sample is almost identical to the slope of unloading line. It is observed that, for the aggregated Abist sample, the slope of unloading line remains unchanged and it is the same for the first unloading-reloading and the final unloading of the sample at the end of compression. In the case of Bioley silt, it is also observed that the slope of the unloading (swelling) line is almost the same in aggregated and reconstituted samples. In this case, however, unloading was performed at the stress level for which the aggregated structure has been already obliterated significantly, as indicated by the convergence of two curves. It is, thus, normal that the two soils have the same behaviour on the unloading path at the end of compression. It is difficult to make a firm conclusion about the influence of aggregation on the unloadingreloading slope based only on these results. However, this influence has been neglected as a simplifying assumption.

\subsection{Pore-scale response}

The experimental technique of neutron tomography and image processing was used here to study the soil structure and its evolution during the oedometric test on the aggregated sample of Abist silt. Neutron tomography is a nondestructive technique for investigating the distribution of neutron attenuating materials [7]. This technique, together with computed tomography algorithms, yields the threedimensional array of the volume of the sample in terms of a local neutron attenuation coefficient. Reconstructed volumes are processed by a sequence of spatial filters, segmentation and morphological operations. Segmentation of the reconstructed volumes into aggregates and the pores between them gives a binary volume of the sample in which these two components can be distinguished.

The concept of double porosity $[3,22]$ in aggregated soil is postulated here for the purpose of soil structure evaluation. The relation among total, macro- and microporosity in aggregated soil reads:

$n=n^{\mu}+n^{\mathrm{m}}$

where $n$ is the total porosity and $n^{\mu}$ and $n^{\mathrm{m}}$ denote the microporosity corresponding to the pores within the aggregates (intra-aggregates) and the macroporosity corresponding to the pores between the aggregates (interaggregate), respectively. Macro- or microporosity, therefore, is the volume of the macro or micropores divided by the whole volume of the soil.

To assess the soil structure evolution during the oedometric compression, neutron tomography of the sample is recorded at different loading steps. The macroporosity of 
the sample at different loading steps is then deduced from the segmented volumes of the sample.

Details of the experimental procedures and discussion of the results are beyond the scope of this paper and are presented elsewhere [13]. The main experimental results, which will be used for the purpose of modelling, will be briefly outlined here.

It is observed that no significant change in macroporosity occurs during the initial stiff domain (stress state lower than the apparent preconsolidation stress) or during unloading-reloading paths. Major changes in macroporosity only occur when the loading stress exceeds the apparent preconsolidation stress. Evolution of the macroporosity represents the changes in soil structure of the aggregated soil. Considering the preconsolidation stress as a yield limit that separates the domains of elastic (recoverable) and plastic (irrecoverable) deformations in soil, it is concluded that the soil structure modifications only happen together with the occurrence of irrecoverable deformations.

Furthermore, the experimental results also reveal that both volumetric and deviatoric deformations should contribute to completely remove the aggregated structure of the sample and to bring it into a fully destructured state.

\section{Constitutive modelling framework}

In this study, the reconstituted (destructured) soil of the same mineralogy as the aggregated soil is selected to be a reference state. From the mechanical point of view, aggregated soils could be embedded in general modelling framework proposed for bonded and structured soils. Following the proposition of Burland [5], properties of the reconstituted soil are called here intrinsic properties and shown by an asterisk in subscript $(*)$. The main idea is to depart from a constitutive model, which can already describe the reconstituted soil behaviour and extend its applicability to aggregated soils. Macroscopic experimental observations are used to build the general constitutive framework for aggregated soils. The experimental evidence at the pore-scale level will then be used in the next section in order to introduce and define the soil structure parameters. The proposed framework is appropriate for constitutive models based on the theory of plasticity with strain hardening.

\subsection{Pre-yield and elastic behaviour}

Atkinson and Bransby [2] showed that, with good approximation, the one-dimensional compression (swelling) and the isotropic compression (swelling) can be assumed to be parallel. However, this assumption is valid for soils for which the coefficient of lateral earth pressure at rest, $K_{0}$, remains constant during virgin compression. The parallelism between the isotropic and oedometric compression lines is a simplifying assumption, which allows interpreting the oedometric results in the isotropic compression plane.

Accordingly, on the basis of the oedometric compression tests presented previously, an idealized isotropic compression curve of reconstituted and double structure soil is shown in Fig. 3a. The intrinsic normal consolidation line (iNCL) represents the normal consolidation line of the reconstituted soil. The virgin consolidation line of the aggregated soil is characterized with an initial stiff behaviour followed by yielding (point A in Fig. 3a).
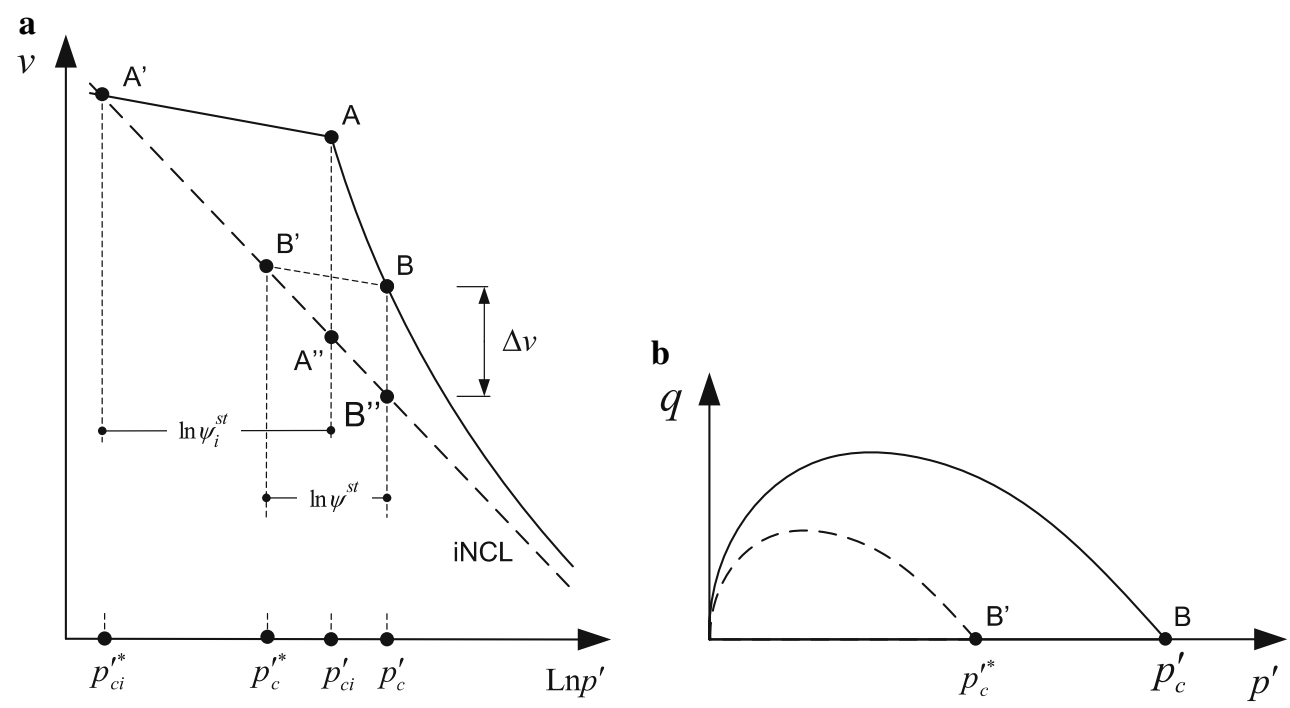

Fig. 3 Comparison of aggregated and reconstituted soils: a isotropic normal consolidation curve; $\mathbf{b}$ extended yield surface 
In this figure, $p_{\mathrm{c}}{ }^{\prime}$ and $p_{\mathrm{ci}}{ }^{\prime}$ are the actual and the initial apparent effective preconsolidation pressure of aggregated soil, respectively. The corresponding intrinsic values for reconstituted soil are denoted by the asterisk (*).

Yielding is determined by the apparent preconsolidation pressure which is identical for samples of the same structure at a similar stress state and stress history. The initial elastic domain is taken into account by introducing an initial yield surface, the size of which is controlled by the initial apparent preconsolidation pressure, $p_{\mathrm{ci}}{ }^{\prime}$, which represents the initial soil structure. As an hypothesis in the present model (and in most of the constitutive models for natural bonded soils) the shape of the yield surface in aggregated soil is assumed to be identical to that corresponding to reconstituted material. The material behaviour inside this yield limit is elastic. Moreover, it is assumed that soil elastic properties are not affected by the structure [15]. Therefore, the elastic behaviour of the aggregated soil is modelled using the reconstituted soil model and elastic properties of the reconstituted soil.

\subsection{Yielding and apparent preconsolidation pressure}

In conventional soil mechanics, the possible states for a reconstituted soil are only those situated on the left side of the normal consolidation line. Experimental results (Sect. 2.1) revealed that the virgin consolidation line of aggregated soil is located to the right side of the normal consolidation line of the reconstituted soil, referred to here as the intrinsic normal consolidation line (iNCL). It means that higher values of mean stress can be applied to the aggregated soil without causing it to yield. In other words, the aggregated soil can sustain higher void ratios at the same stress state ( $\Delta v$ in Fig. 3a).

With the assumption of identical shape of the yield surface for both reconstituted and aggregated soils, this behaviour can be described by a change in size of the yield surface due to an increase of the yield limit (Fig. 3b). Considering the isotropic preconsolidation pressure as the parameter controlling the size of the yield surface, the size of yield surface in aggregated soils is, therefore, determined by the apparent preconsolidation pressure which depends both on stress history and soil structure. As proposed by Gens and Nova [11] for bonded and structured soils, this limit can be considered as an extension of the yield limit of the reconstituted soil at the same void ratio. Accordingly, the apparent preconsolidation pressure of the aggregated soil can be written as:

$p_{\mathrm{c}}^{\prime}=\Psi^{\mathrm{st}} \cdot p_{\mathrm{c}}^{\prime *}$

where $p_{\mathrm{c}}^{\prime}$ is the apparent preconsolidation pressure of the aggregated soil, $p_{\mathrm{ci}}^{\prime *}$ is a reference pressure corresponding to the preconsolidation pressure of the reconstituted soil of the same mineralogy at the same void ratio which represents only the stress history effects. $\Psi^{\text {st }}$ is a function controlling the extension of the yield limit with respect to the reconstituted reference state. This function changes with the evolution of the soil structure and represents the influence of the soil structure on the apparent preconsolidation pressure. The initial value of this function determines the initial soil structure and initial apparent preconsolidation pressure. The mathematical expression of this function, as well as the associated soil structure parameters, will be discussed later.

\subsection{Postyield behaviour and hardening}

When the initial (and subsequent) yield limit is reached, the current yield surface will evolve into a new one according to a hardening rule. Isotropic hardening is postulated here for the sake of simplicity. The yield surface of the reconstituted soil follows a strain-hardening rule, i.e., it changes in size with the occurrence of plastic strains (change in $p_{c}^{\prime *}$ ). Moreover, yielding is associated with structure degradation, expressed by the function $\Psi^{\text {st }}$. Therefore, as the yield limit is reached, the yield surfaces for both reconstituted and aggregated soil change; however, the growth rate is not necessarily the same. The size of the yield locus in aggregated soil is controlled by two phenomena: (1) plastic strain hardening (or softening) of the original model, and (2) softening due to structure degradation. The two phenomena are distinguished in the evolution of the apparent preconsolidation pressure. The evolution rule of the apparent preconsolidation pressure for aggregated soil is obtained from the incremental form of Eq. 2:

$\frac{\mathrm{d} p_{\mathrm{c}}^{\prime}}{p_{\mathrm{c}}^{\prime}}=\frac{\mathrm{d} \Psi^{\mathrm{st}}}{\Psi^{\mathrm{st}}}+\frac{\mathrm{d} p_{\mathrm{c}}^{\prime *}}{p_{\mathrm{c}}^{\prime *}}$

The first term on the r.h.s of this equation represents changes of apparent preconsolidation pressure due to structure effects: softening (or hardening) of the material due to degradation (formation) of structures induced by loading. This term depends on the expression of the structural function $\Psi^{\text {st }}$ and the associated parameters (to be discussed later). The second term represents the strain hardening (or softening) due to stress state and stress history. This term denotes the evolution of the preconsolidation pressure for the reconstituted soil and is, therefore, given by the evolution rule of the original constitutive model with the reconstituted soil properties. With advances in induced structure degradation, the influence of the first term in Eq. 3 decreases and model predictions for aggregated soil become more analogous to those for reconstituted soil. 


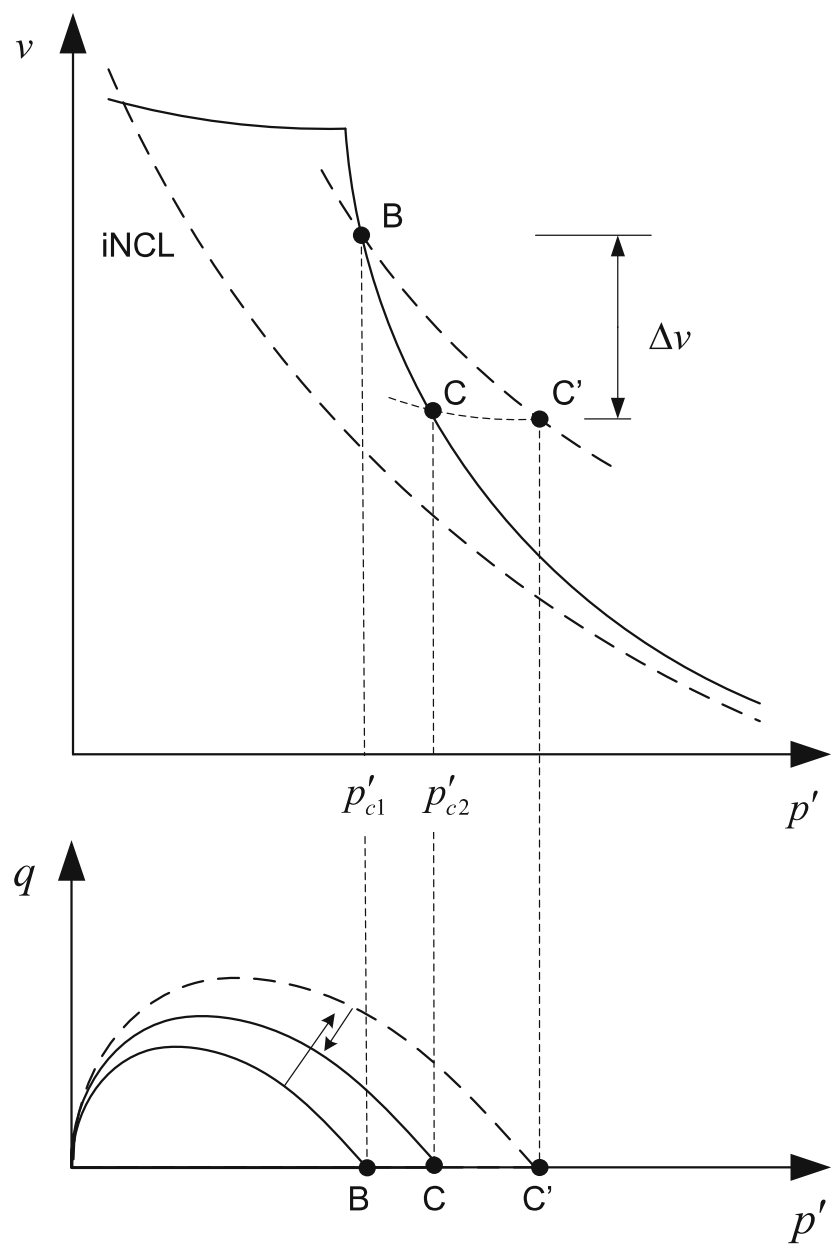

Fig. 4 Evolution of apparent preconsolidation pressure and yield surface in aggregated soils

The two phenomena are schematically depicted in Fig. 4. For point B on the SCL, further isotropic compression brings the soil to point C. However, according to the hardening rule of the reconstituted soil, the apparent preconsolidation (yield limit) should follow a path similar to iNCL and reach point $\mathrm{C}^{\prime}$. Hence, the difference between $\mathrm{C}$ and $\mathrm{C}^{\prime}$ stems from the softening due to structure degradation. On the stress plane $q-p^{\prime}$, the current yield surface passes through point B. The second term on the r.h.s of Eq. 3 is responsible for the enlargement of the yield surface to the one passing through $\mathrm{C}^{\prime}$, while the first term describes the shrinkage of this latter surface to the new yield surface passing through $\mathrm{C}$.

The magnitude of plastic strain, given by plastic multipliers, is determined by satisfying the condition of consistency. The consistency equation requires that a yield criterion be satisfied as long as the material is in a plastic state ( $d f=0$, where $f$ is the yield function). Due to the dependency of yield limit on soil structure, Eq. 2, the consistency equation involves new terms corresponding to the soil structure parameters. This affects the plastic multipliers deduced from this equation. Accordingly, for a given increment of stress, the model yields a higher value of deformation for aggregated soil.

An appropriate choice of flow rule should be based on experimental results, which investigate the effects of structures on the direction of plastic strains. However, for lack of experimental results, it is assumed that the flow rule of the reconstituted soil is valid for the aggregated soil as well. With this hypothesis, the expression of plastic potential is the same for both soils.

\section{Soil structure parameters}

The general constitutive framework proposed here essentially uses the function $\Psi^{\text {st }}$, which appears in the expression of the yield limit, Eq. 2, to include soil structure effects in the model. Proposing a mathematical expression for this function requires, as a first step, the quantification of soil structure as a state parameter of the material. Although macroporosity appears to be a useful measure for evaluating soil structure, it might vary widely for different types of soil. Quantification of soil structure and its degradation requires an internal parameter capable of representing the state of the material with relation to its initial intact condition. For this purpose, the degree of soil structure is introduced here as the ratio of the current macrovoid ratio, defined as $e^{\mathrm{m}}=n^{\mathrm{m}} /(1-n)$, to its initial value at the intact state $e_{i}^{\mathrm{m}}$ :

$R=\frac{e^{\mathrm{m}}}{e_{i}^{\mathrm{m}}}$

Equation 4 defines the degree of soil structure, $R$, as a physical parameter at the pore scale. However, in order for this parameter to contribute to the macroscopic description of the material in a way consistent with the constitutive models, it has to be linked with macroscopic variables of the material.

On the basis of tomography observations, mechanically induced changes in the soil structure can be related to plastic strains, which are identified as a macroscopic variable. Depending on the mechanism of structure degradation, the associated plastic strain might be selected to be isotropic, deviatoric or a combination of both. Isotropic mechanism is mainly associated with isotropic type of loading involving volume change. While, deviatoric mechanism involves distortional deformations and is associated with deviatoric loading. The tomography results suggest that the volumetric plastic strain cannot, by itself, explain the whole process of destructuring. In other words, to reach a fully destructured state, a combination of both isotropic and deviatoric mechanisms is required. 
The parameter $R$ is an internal scaling parameter, which equals unity for an intact aggregated soil with macropores and zero for a fully destructured soil. Any structure degradation, irrespective of its cause, might alter soil structure and consequently the degree of soil structure, $R$. Figure 5 depicts the degree of soil structure for the aggregated sample of Abist silt deduced from the tomography results at different loading steps during the oedometric test plotted versus the associated volumetric plastic strain (only volumetric strains are considered here). The experimental points in this figure are obtained using Eq. 4 in which the values of macrovoid ratio are deduced from the reconstructed tomography volume by image analysis.

This evolution can be well reproduced by a decreasing exponential function of plastic strain:

$R=\exp \left(-\omega \varepsilon^{\mathrm{D}}\right)$

where $\varepsilon^{\mathrm{D}}$ (referred to as destructuring strain) is a general plastic strain which, depending on the loading condition, could be volumetric, deviatoric or a combination of both plastic strains, and $\omega$ is the parameter controlling the rate of structure degradation with plastic deformation. This equation establishes a link between the soil structure and the macroscopic behaviour of the material on the basis on experimental observations at both scales.

Introducing the degree of soil structure as a state parameter to quantify the soil structure, the structural function $\Psi^{\text {st }}$ can now be expressed in terms of this parameter. This function, as given by Eq. 2, represents the

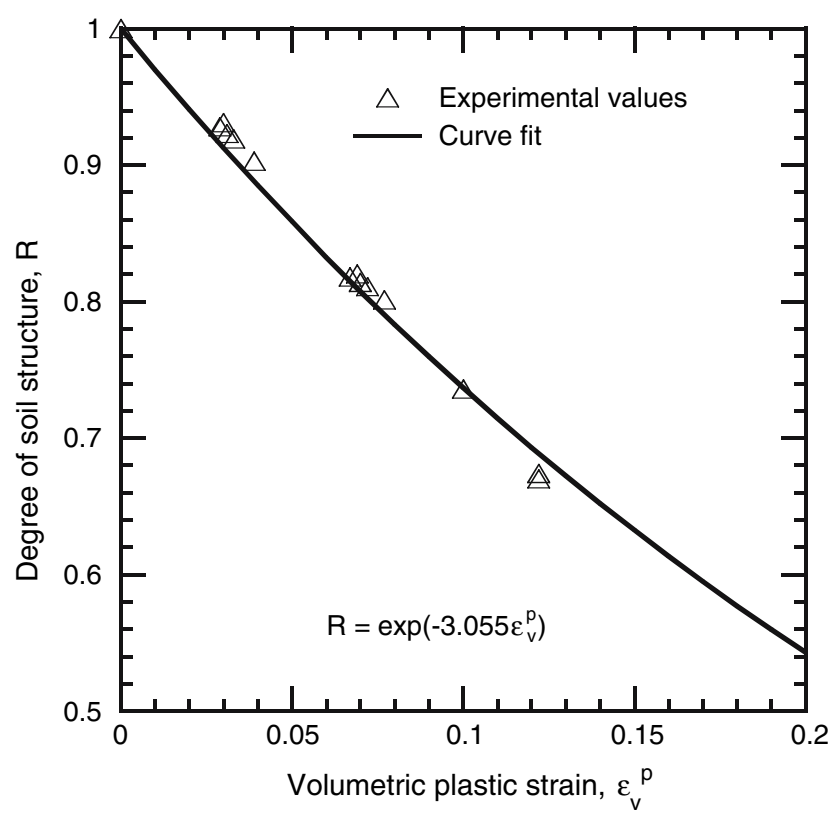

Fig. 5 Degree of soil structure obtained from neutron tomography analysis extra strength of the material due to inter-particle bonding effects. This function should now be expressed in terms of the degree of soil structure, which is a physical parameter of the soil fabric. Such a relation expresses the gain in apparent preconsolidation pressure due to the current state of the aggregated fabric.

According to Eq. 3, the horizontal distance of the two curves in the isotropic compression plane for any specific volume (e.g. $\mathrm{BB}^{\prime}$ in Fig. 3a) is given by $\ln \left(\Psi^{\text {st }}\right)$ with the function $\Psi^{\text {st }}$ corresponding to the current state of aggregated soil. The initial value of this function, $\Psi_{\mathrm{i}}^{\mathrm{st}}$, is a material parameter corresponding to $R=1$. This parameter determines the horizontal separation of the two curves at initial apparent preconsolidation pressure $\left(\mathrm{AA}^{\prime}\right)$. When the soil is fully destructured, there is no extra effect of soil structure and, therefore, the two yield limits are the same $\left(\Psi^{\mathrm{st}}(R)=1\right)$. This is equivalent to the earlier observation that the two compression curves tend to converge at higher values of applied stress.

It was previously observed that at a given value of applied stress, aggregated soil shows a higher void ratio (or specific volume) than reconstituted soil. This is due to the existence of macropores within the aggregated soil.

Therefore, the difference in void ratio between aggregated and reconstituted soil for a given value of $p^{\prime}$ (e.g. $\mathrm{AA}^{\prime \prime}$ in Fig. 3a) for initial apparent preconsolidation pressure must be attributed to macropores. As a result of induced degradation of the structures, the macroporosity decreases and the microporosity, as in reconstituted soils, becomes dominant. Hence, the vertical separation of the two curves in isotropic compression space decreases. At an ultimate state with no macropores, the two curves converge.

Therefore, the relation between the current difference in the void ratio (or specific volume) of aggregated and reconstituted soil in the isotropic plane $\left(\mathrm{BB}^{\prime \prime}\right.$ in Fig. 3a) and its value at initial yielding $\left(\mathrm{AA}^{\prime \prime}\right)$ can be approximated as:

$\frac{\mathrm{BB}^{\prime \prime}}{\mathrm{AA}^{\prime \prime}}=\frac{e^{\mathrm{m}}}{e_{i}^{\mathrm{m}}}=R$

On the other hand, geometrical similarity between triangles $\mathrm{AA}^{\prime} \mathrm{A}^{\prime \prime}$ and $\mathrm{BB}^{\prime} \mathrm{B}^{\prime \prime}$ yields

$\frac{\mathrm{BB}^{\prime \prime}}{\mathrm{AA}^{\prime \prime}}=\frac{\mathrm{BB}^{\prime}}{\mathrm{AA}^{\prime}}=\frac{\ln \left(\Psi^{\mathrm{st}}\right)}{\ln \left(\Psi_{\mathrm{i}}^{\mathrm{st}}\right)}$

Introducing Eq. 4 into Eq. 6 and solving for $\Psi^{\text {st }}$ gives

$\Psi^{\text {st }}=\exp \left[R \ln \left(\Psi_{\mathrm{i}}^{\mathrm{st}}\right)\right]$

The above equation presents $\Psi^{\text {st }}$ at the current state as a function of its initial value and corresponding degree of soil structure. 


\section{Model assessment}

The constitutive framework presented above is applicable to any plastic hardening constitutive model as an extension to aggregated soils. In this study, the proposed developments were applied to the ACMEG model (acronym for advanced constitutive modelling in environmental geomaterials) originally developed for non-structured soils [16]. This model is a strain-hardening model from the Cam Clay family with isotropic and deviatoric plastic mechanisms.

The extended model, named ACMEG-2S, is used here to reproduce the response of the aggregated Bioley silt during oedometric compression. The model uses the material parameters of the reconstituted soil, referred to as intrinsic soil properties and denoted by an asterisk (*). The main material parameters are listed in Table 1.

The model predictions, as well as the experimental results, are plotted in Fig. 6. The reconstituted oedometric curve is first predicted by setting the value of $\omega$ to zero (no destructuring) and of $\Psi_{\mathrm{i}}^{\text {st }}$ to one. The aggregated behaviour is then modelled using the values of the parameters given in Table 1. Due to similarity of Abist and Bioley soil, the value of parameter $\omega$ deduced from tomography results for Abist soil (Fig. 5) is used to simulate the behaviour of Bioley silt. The initial value of $\Psi_{i}^{\text {st }}$ is calculated through comparison between the compression curve of aggregated and reconstituted soil and using Eq. 1 for initial apparent preconsolidation stress.

It is observed that the model prediction is close to the experimental data. These results indicate the model's ability to reproduce the special behaviour of aggregated soils. These results are presented here as a general assessment of the proposed constitutive framework, and are not considered as a validation of the particular constitutive model proposed by the authors. Detailed formulation and validation of this latter one is to be published in a future paper.

\section{Conclusions}

In this study, a general constitutive framework is proposed to describe the mechanical behaviour of aggregated soils.

Table 1 Material properties for dry aggregated Bioley silt

\begin{tabular}{llc}
\hline Parameter & Symbol & Value \\
\hline Intrinsic compression index & $\mathrm{C}_{\mathrm{c}}^{*}$ & 0.267 \\
Intrinsic swelling index & $\mathrm{C}_{\mathrm{s}}^{*}$ & 0.009 \\
Intrinsic angle of friction & $\varphi^{*}$ & 31.8 \\
Rate controlling parameters of & $\omega$ & 3.055 \\
$\quad$ structure degradation & & 35 \\
Initial value of function $\Psi^{\text {st }}$ & $\Psi_{\mathrm{i}}^{\text {st }}$ & 35 \\
\hline
\end{tabular}

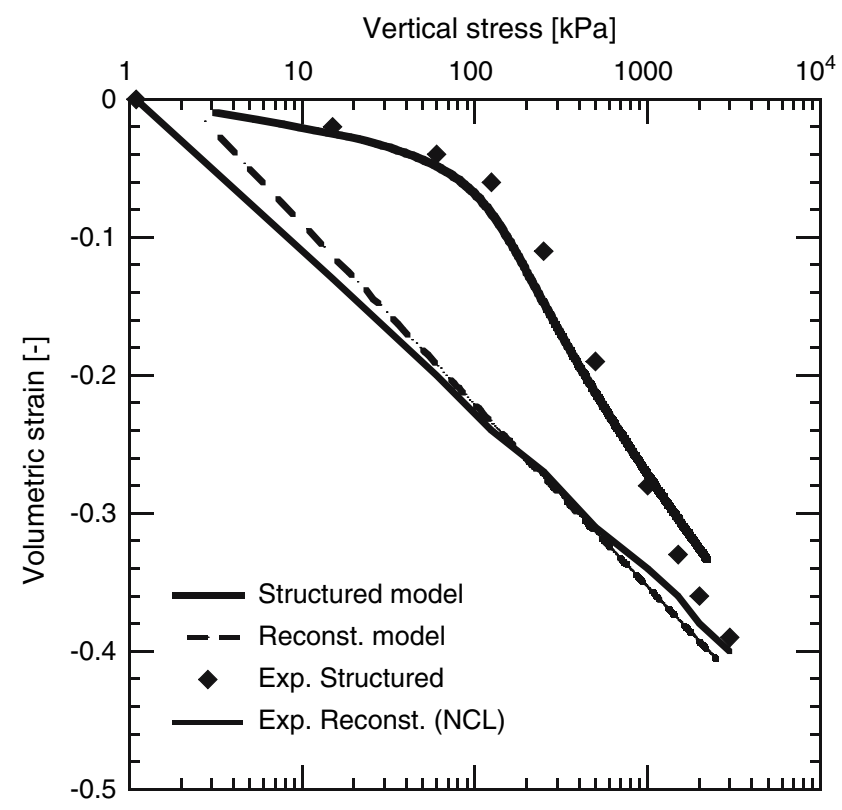

Fig. 6 Prediction of ACMEG-2S constitutive model for aggregated and reconstituted Bioley silt

The proposed framework is a new adaptation of the modelling concept of Gens and Nova [11] to aggregated soils with respect to double porosity fabric. Oedometric compression tests on aggregated samples revealed the existence of an initial stiff behaviour followed by yielding. This phenomenon is similar to induced overconsolidation; however, the nature of the phenomenon is different. The yield limit is, therefore, termed apparent preconsolidation stress (apparent preconsolidation pressure in the isotropic compression plane), which is a function not only of stress state and stress history but also of the particular soil structure. Moreover, the neutron tomography technique is used to evaluate the structure of an aggregated soil sample and its evolution at the pore scale during the oedometric test. These results reveal that significant changes in macroporosity occur only during normal consolidation. It is, therefore, concluded that any major structure evolution is associated with plastic strain. A state parameter, referred to as degree of soil structure, is introduced to quantify the soil structure in terms of macrovoid ratio. On the basis of tomography results, an evolution rule for the degree of soil structure is proposed which relates the changes in soil structure to the plastic strains of the material. On the other hand, the higher void ratio of aggregated soil in comparison to that of the reconstituted soil is attributed to the existence of macropores. Considering this fact in an isotropic compression space, the extra strength components of the aggregated soil are linked to its fabric using the degree of soil structure. This parameter and its evolution rule are then used to introduce a new yield limit for the aggregated soil with a dependency on the soil structure. The proposed 
constitutive framework easily allows any existing constitutive model for reconstituted soil to be extended to aggregated soil.

Acknowledgments This project was funded by EPFL. The authors wish to thank Dr. P. Lehmann for his help in image processing and the partners of this project at ITÖ-ETHZ, Prof. H. Fluehler and Dr. A. Carminati, and at PSI for access to the neutron beam facility, in particular Dr. E. Lehmann and Mr. P. Vöntobel.

\section{References}

1. Al-Mukhtar M (1995) Macroscopic behavior and microstructural properties of a kaolinite clay under controlled mechanical and hydraulic state. In: Alonso EE, Delage P (eds) Unsaturated soils: proceedings of first international conference on unsaturated soils/ UNSAT 95, Paris, pp 3-9

2. Atkinson JH, Bransby PL (1978) The mechanics of soils, an introduction to critical state soil mechanics. McGraw-Hill, London

3. Barrenblatt GI (1963) On certain boundary value problems for the equation of seepage of liquid in fissured rock. J Appl Math Mech 27:513-518 (translation of the Soviet journal, Prikladnaja Matematika i Mekhanika (PMM))

4. Baudet B, Stallebrass S (2004) A constitutive model for structured clays. Géotechnique 54(4):269-278

5. Burland JB (1990) On the compressibility and shear strength of natural clays. Géotechnique 40(3):329-378

6. Callisto L, Rampello S (2004) An interpretation of structural degradation for three natural clays. Can Geotech J 41:392-407

7. Degueldre C, Pleinert H, Maguire P, Lehmann E, Missimer J, Hammer J, Leenders K, Böck H, Townsend D (1996) Porosity and pathway determination in crystalline rock by positron emission tomography and neutron radiography. Earth Planet Sci Lett 140:213-225

8. Delage P, Audiguier M, Cui Y-J, Howat MD (1996) Microstructure of compacted silt. Can Geotech J 33:150-158

9. Gens A, Alonso EE (1992) A framework for the behaviour of unsaturated expansive clays. Can Geotech J 29:1013-1032

10. Gens A, Alonso EE, Suriol J, Lloret A (1995) Effect of structure on the volumetric behavior of a compacted soil. In: Alonso EE,
Delage P (eds) Unsaturated soils: proceedings of first international conference on unsaturated soils/UNSAT 95, Paris, pp 8388

11. Gens A, Nova R (1993) Conceptual bases for a constitutive model for bonded soils and weak rocks. In: Anagnostopoulos A, Schlosser F, Kalteziotis N, Frank R (eds) Geotechnical engineering of hard soils—soft rocks. Balkema, Rotterdam, pp 485-494

12. Kavvadas M, Amorosi A (2000) A constitutive model for structured soils. Geotechnique 50(3):263-273

13. Koliji A, Lehmann P, Vulliet L, Laloui L, Carminati A, Vontobel P, Hassanein R (2007) Structure changes during the mechanical loading of a soil with double porosity (submitted)

14. Koliji A, Laloui L, Cusinier O, Vulliet L (2006a) Suction induced effects on the fabric of a structured soil. Transp Porous Media 64(2):261-278

15. Koliji A, Vulliet L, Laloui L, Carminati A, Kaestner A, Flühler H, Lehmann P, Hassanein R, Lehmann E, Vontobel P (2006b) Structure degradation of dry aggregated soils: experimental evidence and model formulation. In: Miller GA, Zaparat CE, Houston SL, Fredlund DG (eds), Fourth international conference unsaturated soils, UNSAT06, USA, pp 2174-2185

16. Laloui L, Cekerevac C, François B (2005) Constitutive modelling of the thermo-plastic behaviour of soils. Rev Eur Génie Civ 9(5-6):635-650

17. Leroueil S, Vaughan PR (1990) The general and congruent effects of structure in natural soils and weak rocks. Géotechnique 40(3):467-488

18. Mitchell JK (1993) Fundamentals of soil behavior. Wiley, London

19. Nova R, Castellanza R, Tamagnini R (2003) A constitutive model for bonded geomaterials subject to mechanical and/or chemical degradation. Int $\mathrm{J}$ Numer Anal Methods Geomech 27(9):705-732

20. Rouainia M, Wood DM (2000) A kinematic hardening constitutive model for natural clays with loss of structure. Geotechnique 50(2):153-164

21. Sánchez M, Gens A, Guimaraes LD, Olivella S (2005) A double structure generalized plasticity model for expansive materials. Int J Numer Anal Methods Geomech 29(8):751-787

22. Warren JR, Root PJ (1963) The behaviour of naturally fractured reservoirs. Soc Petrol Eng J 3(3):245-255 